\title{
A Nondestructive Method for Measurements of Complex Permittivity of Microwave Dielectric Substrate Materials
}

\author{
Fei Zhao ${ }^{1, a}{ }^{*}$, Pei $\mathrm{Xu}^{1, \mathrm{~b}}$, Changtao Sha ${ }^{1, \mathrm{c}}$, Wenfeng Wang ${ }^{1, \mathrm{~d}}$ and Jinsong Kan ${ }^{1, \mathrm{e}}$ \\ ${ }^{1}$ China Electronic Standardization Institute, Beijing 100176, P.R. China. \\ azhaofei@cesi.cn, ${ }^{b} x u p e i @ c e s i . c n,{ }^{\circ}$ shact@cesi.cn, ${ }^{\mathrm{d}}$ wangwf@cesi.cn, ${ }^{\mathrm{k}} \mathrm{kanjs} @ \mathrm{cesi.cn}$
}

Keywords: dielectric substrates, microwave measurement, permittivity measurement, nondestructive testing, cavity resonator.

Abstract. A nondestructive and accurate measurement method and related apparatus based on the split-cylinder resonator techniques is descried in this paper. The dielectric constants and loss tangents of the flat substrate materials are suitable to be characterized and then calculated by software programmed according to a rigorous mode match analysis of the $\mathrm{TE}_{011}$ mode. The dielectric properties of some typical substrate materials have been measured, and measurement uncertainties are also given in this paper. In addition, the effect of sample thickness on the dielectric properties is also investigated. These results demonstrate that this technology is capable of accurately characterizing the dielectric properties of flat substrate materials versus frequency by changing the sample thickness.

\section{Introduction}

The dielectric substrate materials used in patch antennas and RF/microwave circuits demand relatively low dielectric constant and low dielectric loss. The design and fabrication of patch antennas and microstrip circuits often require accurate measurements of the substrate dielectric properties at microwave frequencies. And for online quality control in substrate manufacturing and processing, it is also necessary to develop a nondestructive method for dielectric measurement.

There are a large number of methods for determining the permittivity of a single homogeneous sample or the effective "bulk" properties of a layered material. These methods include cavity resonators, split-post dielectric and magnetic resonators, whispering-gallery resonators, transmission-line and waveguide techniques, etc.. However, most of these methods generally require the sample to be machined accurately into a suitable geometry to fit the resonant cavity. That is often difficult for some materials, such as ceramics, and certainly undesirable for nondestructive testing. For measurements of the complex permittivity of the flat substrate materials in a nondestructive way, the open-end coaxial probe method [1-2] is a nondestructive and broadband technique, whereas the split-cylinder resonator method is as the most accurate method. Therefore, the later method is generally preferred for accurate dielectric measurement of the substrate materials.

In this paper, a split-cylinder resonator method and related apparatus is descried. This method was first developed by Kent [3]. Fang and Linton et al. [4-5] suggested a developed apparatus and made a systematic analysis based on a rigorous mode match model. Herein, we construct a measurement apparatu, of which the nominal resonant frequency of $\mathrm{TE}_{011}$ mode is $10 \mathrm{GHz}$. The complex permittivity is then extracted by considering only the resonant mode in the mode match analysis, with the help of a small calculation program based on the rigorous mode match method. A series of substrate materials with different dielectric constant values have been measured at multiple frequencies. The measurement results are presented and discussed in this paper.

\section{Measurement Techniques}

Measurement Theory. The present method aims at measuring the complex permittivity of the microwave substrate materials in a nondestructive way. A full-wave analysis of the split cylinder resonator was investigated by Janezic et al.[6]. The model includes the resonant mode plus evanescent modes in the cylindrical cavity region. For more details, the reader can refer to ref [6]. 
Measurement System. We construct a measurement apparatus, of which the nominal resonant frequency of $\mathrm{TE}_{011}$ mode is $10 \mathrm{GHz}$. The apparatus is shown in Fig. 1. The resonant frequency is obtained by measuring the $\mathrm{S}$ parameters versus frequency from the Agilent 8363B network analyzer. Software was programmed for data acquisition and computations. The flowchart of the measurement calculation software is shown in Fig. 2, which also indicates the whole procedure of the complex permittivity measurement.

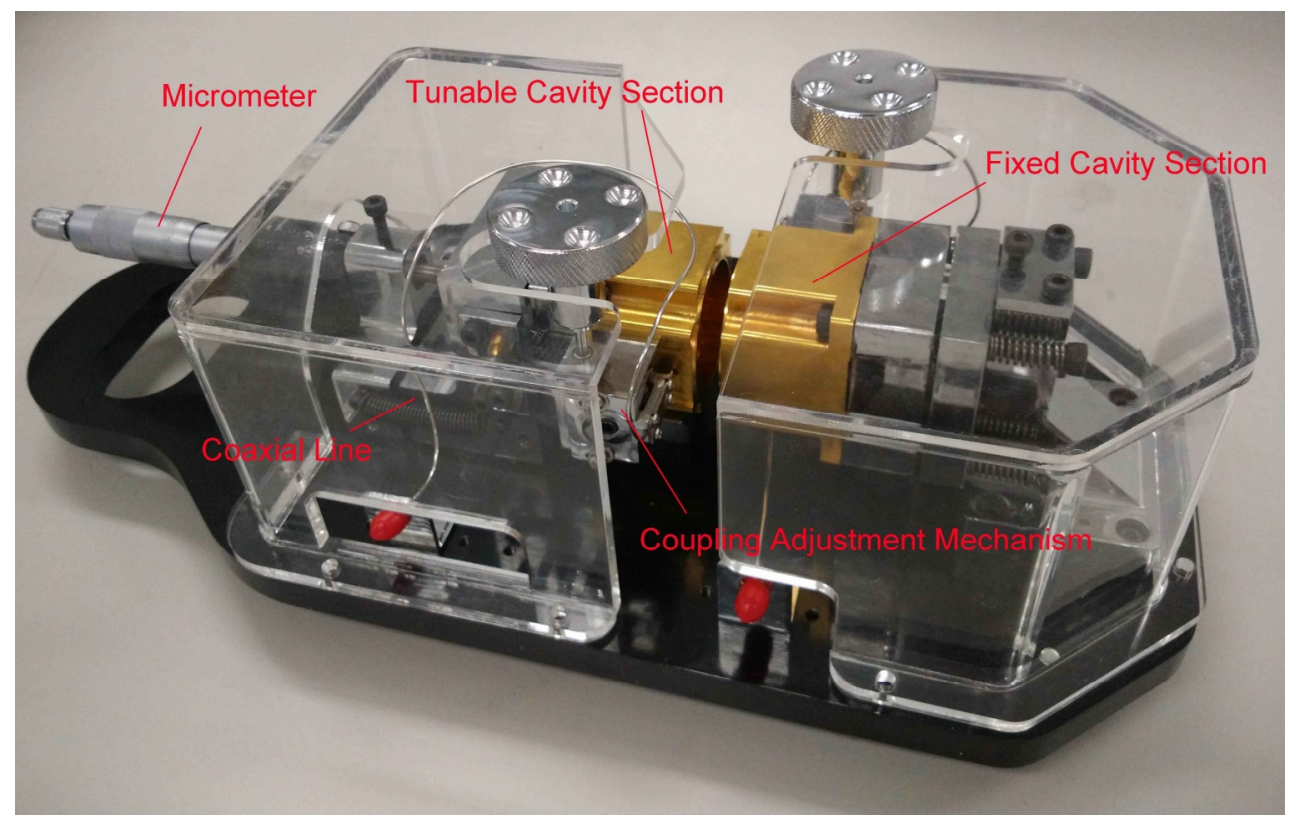

Fig. 1. Experimental system of the split-cylinder resonator method.

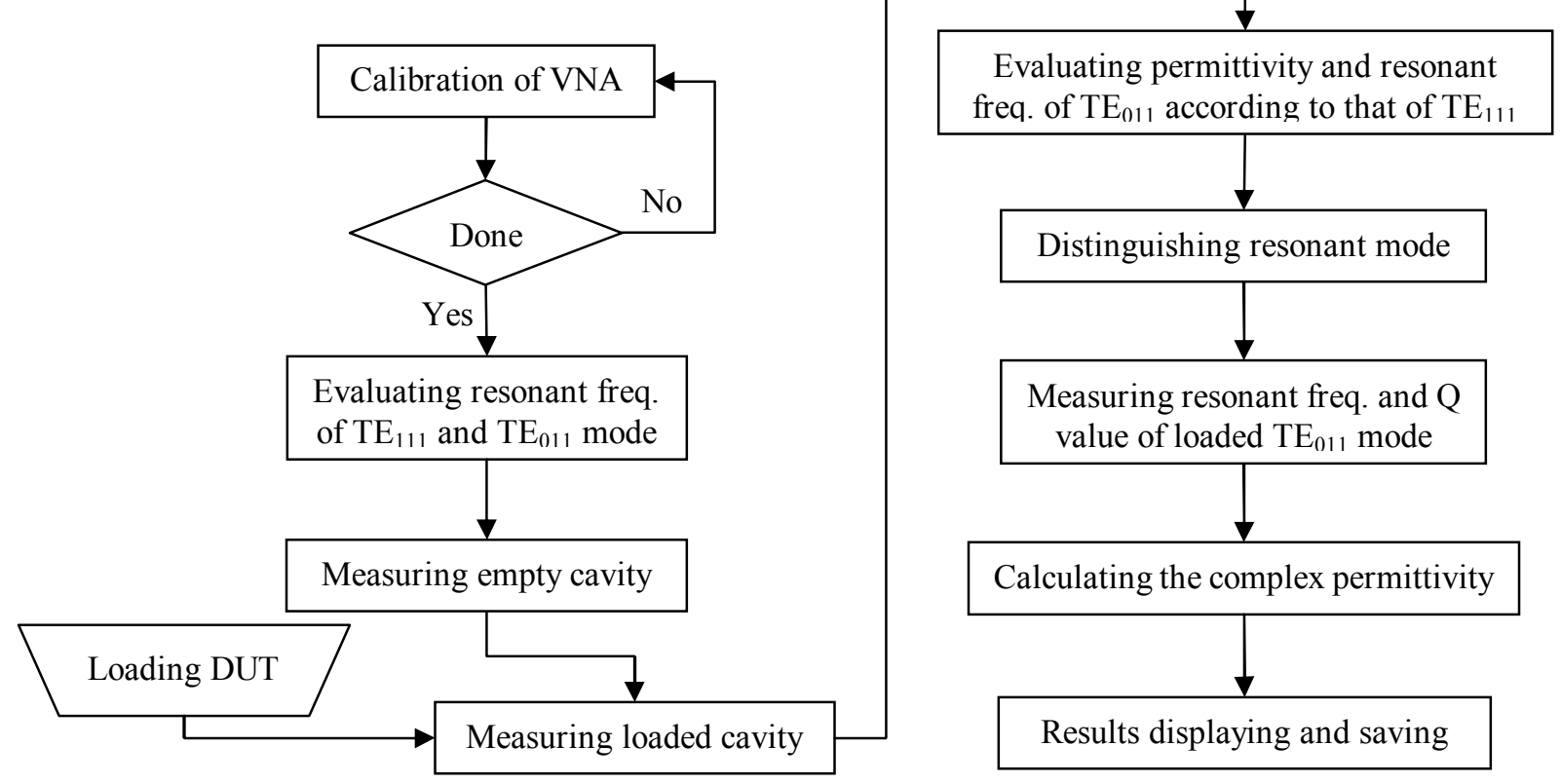

Fig. 2. Procedure of the complex permittivity measurement.

Measurement Uncertainty Analysis. The uncertainty $\varepsilon_{\mathrm{r}}$ includes errors resulting from the uncertainties of the resonant frequency, sample thickness, cavity diameter and length. It can be expressed by the following equation:

$$
u_{c}^{2}\left(\varepsilon_{\mathrm{r}}\right)=\left(\frac{\partial \varepsilon_{\mathrm{r}}}{\partial f}\right)^{2} u^{2}(f)+\left(\frac{\partial \varepsilon_{\mathrm{r}}}{\partial a}\right)^{2} u^{2}(a)+\left(\frac{\partial \varepsilon_{\mathrm{r}}}{\partial L}\right)^{2} u^{2}(L)+\left(\frac{\partial \varepsilon_{\mathrm{r}}}{\partial d}\right)^{2} u^{2}(d)
$$


The partial differentials of $\varepsilon_{\mathrm{r}}$ in equation (1) can be numerically calculated by giving uncertainties: $\Delta f$, $\Delta d, \Delta L, \Delta a$ respectively; where $f, d, L, a$, are the resonant frequency of the $\mathrm{TE}_{011}$ mode, sample thickness, cavity length and cavity radius respectively. It should be mentioned that this expression takes only the $\mathrm{TE}_{011}$ mode into account.

\section{Results and Discussion}

Four typical substrate samples with different nominal dielectric constant have been measured using the split cylindrical resonator. The samples, with a diameter larger than that of the cylindrical cavity, were inserted into the gap of the split cylindrical resonator. Fig. 3 shows an example of the curves from which the resonant frequency $f_{\mathrm{r}}$ and $-3 \mathrm{~dB}$ bandwidth of the resonant curves of $\mathrm{TE}_{011}$ modes could be extracted, and, thus, the dielectric constants $\left(\varepsilon_{\mathrm{r}}\right)$ and loss tangents $(\tan \delta)$ of the samples can be extracted by the software mentioned above.

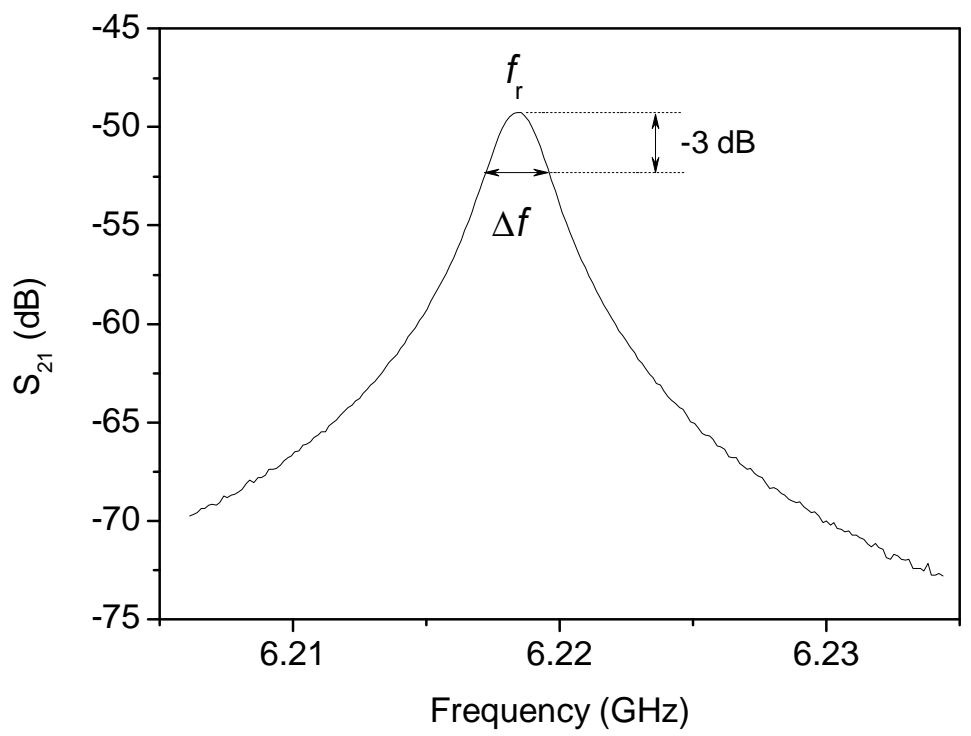

Fig. 3. Resonance curves measured by K20 ceramic sample loaded resonator.

The measurement results and their corresponding uncertainties, as well as the referenced values from Ref [7] and the Hakki-Coleman dielectric resonator method are listed in Table 1. It can be seen that the present results obtained by the split-cylinder resonator method are in agreement with the referenced values. This method can be applied in low-loss substrates with a wide range of dielectric constant and frequencies.

Table 1 The dielectric constants $\left(\varepsilon_{\mathrm{r}}\right)$ and loss tangents $(\tan \delta)$ of selected samples.

\begin{tabular}{|c|c|c|c|c|c|c|}
\hline \multirow[b]{2}{*}{ Sample } & \multicolumn{3}{|c|}{ Measured } & \multicolumn{3}{|c|}{ Referenced } \\
\hline & $\begin{array}{c}\text { Frequency } \\
{[\mathrm{GHz}]}\end{array}$ & $\varepsilon_{\mathrm{r}}$ & $\tan \delta$ & $\begin{array}{c}\text { Frequency } \\
{[\mathrm{GHz}]}\end{array}$ & $\varepsilon_{\mathrm{r}}$ & $\tan \delta$ \\
\hline \multirow{3}{*}{ Fused silica } & 9.6811 & 3.81 & $1.72 \times 10^{-4}$ & \multirow{3}{*}{9.504} & \multirow{3}{*}{3.833} & \multirow{3}{*}{$1.39 \times 10^{-4}$} \\
\hline & 9.3732 & 3.82 & $1.43 \times 10^{-4}$ & & & \\
\hline & 8.6029 & 3.80 & $1.86 \times 10^{-4}$ & & & \\
\hline \multirow{3}{*}{ K20 ceramic } & 7.6294 & 19.66 & $0.93 \times 10^{-4}$ & \multirow{3}{*}{6.028} & \multirow{3}{*}{19.71} & \multirow[t]{3}{*}{$0.77 \times 10^{-4}$} \\
\hline & 6.2124 & 19.82 & $0.92 \times 10^{-4}$ & & & \\
\hline & 4.8295 & 19.47 & $0.98 \times 10^{-4}$ & & & \\
\hline
\end{tabular}

The uncertainties for all the samples were analyzed numerically by solving the solutions to the partial derivatives in (1). As an example, the error sources along with their contribution to the 
combined standard uncertainty in for one of the samples measured (K20) are illustrated in Table 2. The largest contributions of uncertainty come from the uncertainty in the cylindrical waveguide radius and sample thickness.

Table 2 Error Sources along with their contributions towards the combined standard uncertainty in dielectric constants $\left(\varepsilon_{\mathrm{r}}\right)$ for samples of fused silica and K20 ceramic.

\begin{tabular}{|c|c|c|c|c|}
\hline \multirow{2}{*}{$\begin{array}{c}\text { Parameter } \\
\text { (PRM.) }\end{array}$} & \multicolumn{2}{|c|}{ Fused silica } & \multicolumn{2}{c|}{ K20 ceramic } \\
\cline { 2 - 5 } & PRM. $\pm \Delta$ PRM. & $\Delta \varepsilon_{\mathrm{r}}$ & PRM. $\pm \Delta$ PRM. & $\Delta \varepsilon_{\mathrm{r}}$ \\
\hline Cylindrical Waveguide Radius $a(\mathrm{~mm})$ & $19.14 \pm 0.040$ & 0.019 & $19.14 \pm 0.040$ & 0.02 \\
\hline Cylindrical Waveguide Length $L(\mathrm{~mm})$ & $25.40 \pm 0.041$ & 0.0013 & $25.40 \pm 0.041$ & 0.0001 \\
\hline Sample Thickness $d(\mathrm{~mm})$ & $0.955 \pm 0.006$ & 0.013 & $1.012 \pm 0.008$ & 0.08 \\
\hline Resonant Frequency $f_{0}(\mathrm{GHz})$ & $9.3581 \pm 0.00003$ & 0.00017 & $6.2184 \pm 0.00003$ & 0.0002 \\
\hline $\begin{array}{c}\text { Measurement Repeatablity } \\
\text { Experimental standard Deviation } s(x)\end{array}$ & --- & 0.002 & - & 0.056 \\
\hline Combined Standard Uncertainty $u_{c}$ & \multicolumn{2}{|c|}{0.023} & \multicolumn{2}{c|}{0.10} \\
\hline $\begin{array}{c}\text { Expanded Measurement Uncertainty } U \\
(k=2)\end{array}$ & \multicolumn{2}{|c|}{$\begin{array}{c}0.046 \\
\left(U_{\text {rel }}=1.2 \%\right)\end{array}$} \\
\hline
\end{tabular}

\section{Summary}

We constructed a split-cylinder resonator measurement setup to characterize the dielectric constants and loss tangents of the flat substrate materials. Measurement results of two typical substrate samples with different thickness show that this nondestructive method is a competitive method for dielectric properties characterization of the low-loss substrates.

\section{References}

[1] D.K. Misra, A quasi-static analysis of open-end coaxial lines, IEEE Trans. Microw. Theory Tech. 35 (1987) 925-928.

[2] D.K. Misra, On the measurement of the complex permittivity of materials by an open-end coaxial probe, IEEE Microw. Guid. Wave Lett. 5 (1995) 161-163.

[3] G. Kent, Nondestructive permittivity measurement of substrates, IEEE Trans. Instrum. Meas. 45 (1996) 102-106.

[4] X.Y. Fang, D. Linton, C. Walker and B. Collins, Non-destructive characterization for dielectric loss of low permittivity substrate materials, Meas. Sci. Technol. 15 (2004) 747-757.

[5] X.Y. Fang, D. Linton, C. Walker and B. Collins, A tunable split resonator method for nondestructive permittivity characterization, IEEE Trans. Instrum. Meas. 53 (2004) 1473-1478.

[6] M.D. Janezic and J. Baker-Jarvis, Full-wave analysis of a split-cylinder resonator for nondestructive permittivity measurements, IEEE Trans. Microw. Theory Tech. 47 (1999) 2014-2020.

[7] M.D. Janezic, Nondestructive relative permittivity and loss tangent measurements using split-cylinder resonator, Ph.D thesis, Univ. of Colorado, 2003. In Chapter 6. 